Décadrages Décadrages

cınéma, à travers champs Cinéma, à travers champs

10 | 2007

La trilogie de Dieu de João César Monteiro

\title{
Quand la fiction familiale intègre le dispositif de l'entretien : l'effet documentaire dans Mon frère se marie
}

Alain Boillat

\section{OpenEdition}

Journals

Édition électronique

URL : http://journals.openedition.org/decadrages/424

DOI : $10.4000 /$ decadrages. 424

ISSN : 2297-5977

Éditeur

Association Décadrages

Édition imprimée

Date de publication : 10 avril 2007

Pagination : 96-109

ISBN : 978-2-9700582-5-0

ISSN : 2235-7823

Référence électronique

Alain Boillat, "Quand la fiction familiale intègre le dispositif de l'entretien : l'effet documentaire dans

Mon frère se marie », Décadrages [En ligne], 10 | 2007, mis en ligne le 10 décembre 2012, consulté le 02 mai 2019. URL : http://journals.openedition.org/decadrages/424; DOI : 10.4000/decadrages.424 


\section{Quand la fiction familiale intègre le dispositif \\ de l'entretien: l'effet documentaire dans}

\section{Mon frère se marie}

\section{par Alain Boillat}

Remerciements : Adrian Blaser, Séverine Graff

1 Alors que certains recenseurs suisses passent totalement cet aspect sous silence (par exemple Mathieu Loewer, "Noces helvétiques en demi-teinte", Le Courrier, 7.10.2006, p. 23, ou Jean-Louis Kuffer, "Les cabossés de la vie", 24 Heures, 28.09.2006), seul Norbert Creutz prête une grande attention à ce qu'il qualifie de "splendide dispositif de mise en abyme", tout en concluant que "la tension documentaire-fiction devient plus théorique que vraiment réalisée" ("Mariage à I'helvétique", Le Temps, 4.10.2006, p. 33). Pour le chroniqueur du Corriere del Ticino, la technique pseudodocumentaire "semble un peu forcée" ("Una familia unita per il quieto vivere", 9.08.2006, p. 22), alors que Thierry Jobin, dans un article fort dépréciatif qui se fait l'écho de la déception ressentie par une bonne part de la presse helvétique et du public du Festival de Locarno (probablement en raison des attentes démesurées qui avaient été placées en Suisse dans le film), compte parmi les éléments qui "ne marche[nt] pas tout à fait " "la tension entre fiction et documentaire lorsque les personnages sont interviewés face caméra ", jugeant que ces moments à son sens insuffisamment exploités semblent "avoir trop peur de lâcher le réel" ("Divorce avec Mon frère se marie", Le Temps, 9.08.2006, p. 25). On notera qu'en France, ni L'Humanité (31.01.07), ni Le Nouvel Observateur (1.02.07), ni Positif ( ${ }^{\circ} 552$, février 2007), ni Telerama $\left(n^{\circ} 2977,3.02 .07\right)$ n'évoquent le dispositif de l'entretien, dont on trouve cependant une brève mention (entre parenthèses) dans un article de Gilles Renault ("Désunis par les liens du mariage ", Libération, 31.01.07).
Le premier long métrage de fiction réalisé par Jean-Stéphane Bron, Mon frère se marie (2006), présente une particularité qui a été relativement peu commentée (voire peu appréciée) par les critiques à la sortie du film¹, mais sur laquelle il me paraît utile de revenir, dans la mesure où ses implications touchent autant à des questions liées à la théorie de la fiction au cinéma qu'à l'économie narrative du film: il s'agit de l'insertion d'entretiens dans lesquels les personnages, filmés frontalement, apparaissent dans des plans fixes. Ces fragments semblent détachés de la continuité visuelle du reste du film, l'unique «raccord» qu'ils occasionnent relevant précisément d'un type de "saute» que Roger Odin identifie dans le film de famille, où l'on trouve fréquemment «deux plans consécutifs présentant un cadrage identique du même décor et entre lesquels les per-

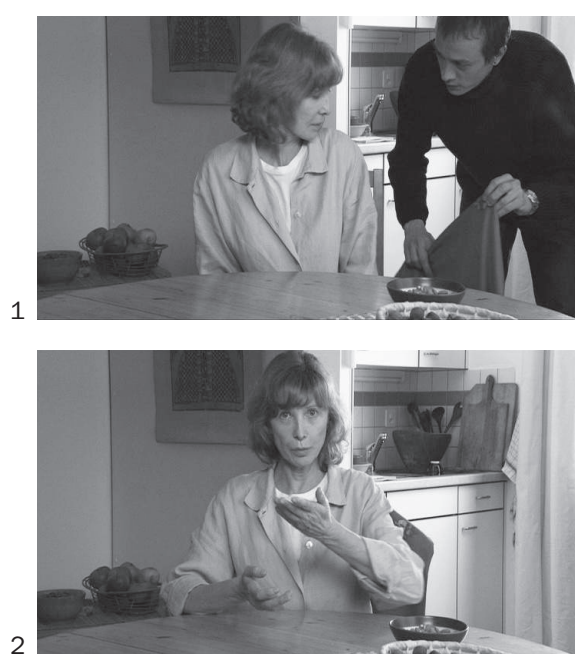


sonnages ont bougé» $\mathbf{2}$; nous verrons que le film de Bron fait un usage productif de la référence à cette pratique non professionnelle. De façon plus générale, la démarche qui consiste à interroger des gens situés face à la caméra confère à ces instants la facture traditionnellement associée aux films dits de "reportage", c'est-à-dire à une pratique de type documentaire. Or Mon frère se marie est indiscutablement un film fictionnel - Jean-Luc Bideau y interprète par exemple un ancien patron désormais au chômage. En outre, bien que ce film soit majoritairement régi par les codes de la comédie, les intermèdes ne sont pas exploités en tant que ressorts d'une complicité amusée avec le public ${ }^{3}$, mais sont présents sur l'ensemble du film, qui ne comprend pas moins de vingt-six occurrences de ce type d'images (voir le tableau en annexe) ${ }^{4}$. Les plans issus de ce dispositif singulier et rigide acquièrent dès lors une fonction de ponctuation, et instaurent une distance par rapport à l'histoire qui, si l'on fait exception de ces intermèdes, est racontée de façon linéaire et traditionnelle: le film narre comment une famille désunie se reconstitue artificiellement lors du mariage du fils adoptif afin de cacher à la mère biologique, venue du Vietnam pour l'occasion (accompagnée d'un oncle), la réalité de la situation familiale des parents suisses. Dans les moments d'entretien par contre, cette position en retrait et le ton de la confession ne sont pas sans évoquer certaines représentations cinématographiques de procès, notamment lorsqu'elles reposent sur un modèle polyphonique (du type de Rashômon de Kurosawa, 1950). Comme le spectateur ne tarde pas à l'apprendre (insert $\left.n^{\circ} 9\right)^{5}$, les protagonistes de Mon frère se marie interviennent de manière rétrospective sur l'histoire, ce qui provoque selon l'emplacement de ces inserts des effets d'annonce, de reprise ou de complémentarité. On pourrait penser, pour utiliser un célèbre exemple helvétique, aux scènes de la pièce Andorra (Max Frisch, 1961) dans lesquelles les différents habitants du village viennent s'exprimer à la barre. Toutefois - et c'est là un aspect essentiel qui fait la richesse de l'usage du procédé dans Mon frère se marie -, les personnages du film de Bron ne s'expriment nullement dans un palais de justice, mais répondent aux questions d'un membre de la famille qui se trouve derrière la caméra, Jacques (Cyril Troley), dans un contexte tout à fait quotidien. La question d'une éventuelle énigme à résoudre subsiste de façon sous-jacente, mais se voit déplacée dans le cadre d'"un film de famille», ce qui redéfinit totalement les enjeux et la visée des entretiens.

\section{Un dispositif qui crédibilise les personnages de fiction}

Les différents personnages interviewés sont la mère (Claire : Aurore Clément) et le père (Michel : Jean-Luc Bideau) de Jacques, sa sœur Catherine
2 Roger Odin, "Le film de famille dans l'institution familiale" dans Roger Odin (éd.), Le Film de famille. Usage privé, usage public, Méridiens Klincksieck, Paris, 1995, p. 30. En ce qui concerne l'emplacement de ces sautes, voir les occurences de la mention "saute temporelle, identité de cadre" dans l'annexe du présent article.

3 Tel est par exemple le cas du prologue de The Incredibles (Les Indestructibles, Brad Bird, 2004), dans lequel différents personnages de synthèse - indice évident du fort degré de fictionalité de l'univers représenté - se confient à la caméra dans un pastiche d'interview télévisée (dont l'image adopte d'ailleurs provisoirement le format). II est à noter que, à l'instar de Mon frère se marie, le film de Bird est une fiction familiale.

4 Le repérage des plans d'entretiens comprend la retranscription de répliques et certaines précisions dont je ne fais pas toujours état dans l'article pour des raisons de place. La numérotation utilisée dans le texte renvoie à cette annexe.

5 Notons que dans l'intervalle séparant les premières images de cette indication temporelle ("jusqu'à ce mariage"), le spectateur peut être amené à conférer une motivation strictement diégétique au tournage effectué par le fils, Jacques: les images qui sont en train d'être tournées pourraient être destinées à une projection conçue comme une animation pour la cérémonie de mariage. 
6 La réflexivité du film (on y fait référence à la présence de la caméra, que les protagonistes fixent continûment) incite le public à l'associer à des productions - dont le film de Bron se distingue toutefois, ainsi qu'on le verra par la suite - qui refusent la transparence pour s'afficher en tant que produits, cela généralement pour attester la réalité de ce qui est montré. En ce sens, le spectateur est enjoint par le dispositif des entretiens à construire un "énonciateur réel de la production" au sens de Roger Odin (De la fiction, DeBoeck, Bruxelles, 2000, pp. 53-61). Ce paratexte filmique de non-fiction permet alors de renforcer la suspension volontaire de l'incrédulité qu'exige le régime fictionnel. Informé du fait qu'il s'agit d'un tournage, le spectateur adhère d'autant plus aux actions et aux paroles du film. Ce que Christian Metz dit de la "voix off" concerne également l'interview dans Mon frère se marie: "par la distance qu'elle met entre l'action et nous, elle conforte notre sentiment de n'être pas dupes de cette action; ainsi rassurés (derrière le rempart), nous pouvons nous permettre d'en être un peu plus dupes (Le signifiant imaginaire, Christian Bourgeois, Paris, 1993 [1977], pp. 100-101).

7 Ainsi un critique affirme-t-il par exemple que l'"on touche carrément au documentaire lorsque les personnages s'expriment dans un film tourné par l'un des membres de la famille" ("Zoom sur une famille recomposée", Le Nouvelliste, 4.10.2006, p. 37).

$\mathbf{8}$ Le spectateur sait qu'il n'assiste pas à un documentaire, mais cette référence assure la cohérence et la vraisemblance de la diégèse. Philippe Hamon et Roland Barthes ont montré pour la littérature combien cette démarche réaliste repose sur une construction visant à produire un effet donné (voir Littérature et réalité, Seuil, Paris, 1982).

9 Roger Odin, "Le film de famille dans l'institution familiale", op. cit., p. 28.

$10 / d .$, p. 27.
(Delphine Chuillot), son frère adoptif Vinh (Quoc Dung Nguyen) et, à une seule occasion, Sarah (Michèle Rohrbach), l'épouse de ce dernier. La mise en scène de ces séquences d'entretien vise avant tout à se faire oublier, c'est-à-dire que, paradoxalement ${ }^{6}$, elle s'affiche en suggérant constamment la présence du dispositif de la prise de vue et en imitant les caractéristiques d'un tournage improvisé (moments creux, aléas, hésitations des locuteurs, etc.). La référence à un style associé aux pratiques documentaires 7 est réinvestie au profit d'un «effet de réel» qui crédibilise l'univers dépeint ${ }^{8}$. L'ouverture du film, où les cartons du générique alternent avec des fragments d'interview, est symptomatique de cet effet: lorsque Jacques ramasse le pull-over de sa mère, les voix sont chuchotées, comme si ces deux «personnes» parlaient des coulisses, d'un horsfilm, comme si le cinéaste avait conservé les amorces que l'on visionne dans les rushes mais que l'on coupe au montage. Aussi, le spectateur a l'impression de surprendre ces deux êtres à un moment quelconque de leur existence, qui plus est dans un cadre tout à fait banal - la cuisinière à l'arrière-plan ne manquant pas de renvoyer à une représentation fort stéréotypée des fonctions attribuées à la mère (fig. 1). L'ouverture du film ne semble pas en être une (au sens rhétorique du terme), tant l'action débute in medias res: en cela, Mon frère se marie emprunte la forme canonique du «film de famille», film «ouvert aux deux bouts» qui «apparaît comme voué à une incomplétude définitive» 9 . Rien de plus stratégique bien sûr que cette apparente absence de discursivité, puisqu'en fait cette esthétique du fragment est mise au service d'une logique proprement «textuelle». Toutefois, cet effet de captation involontaire - selon la logique diégétique, plus personne ne se trouve derrière l'appareil de prise de vue dès lors que Jacques est dans le champ - donne l'impression que les actions montrées n'obéissent à aucune mise en scène. Lors de la seconde occurrence d'un plan d'entretien, la mère interroge son fils sur le dispositif, lui demandant où elle doit regarder et comment se déroulera l'entretien (fig. 2). On notera avant tout qu'elle évoque la question du destinataire des images. Le fils lui répond significativement "ça reste entre nous", renvoyant de manière fictive (ainsi que le constate le spectateur du film assis dans la salle de cinéma) au mode de consommation du «film de famille», un type de production que la plupart des théoriciens définissent en fonction des circonstances de son visionnement à l'intérieur de l'institution familiale - pour Odin, il s'agit d'«un film (ou [d'] une vidéo) réalisé/e par un membre d'une famille à propos de personnages, d'événements ou d'objets liés d'une façon ou d'une autre à l'histoire de cette famille et à usage privilégié des membres de cette famille»10. Ces conditions matérielles, thématiques et communication- 
nelles sont bien celles qui sont postulées par la démarche de Jacques, ainsi que dans la réponse qu'il donne à sa mère. Par contre, peu après, il ressort d'une conversation avec sa sœur qu'il n'a pas dit la vérité à sa mère (voir le dialogue de l'insert $\mathrm{n}^{\circ} 3$ ).

Le filmage frontal des locuteurs (fig. 3 et 4) implique l'omniprésence des regards à la caméra, qui introduisent un régime fondamentalement différent du reste du film (la première question de Claire est significativement: "je te regarde ou je regarde dans la caméra?»), où la diégèse s'autonomise totalement par rapport au tournage, auquel on ne fait jamais allusion. Par contre, dans le prologue et les intermèdes, l'acte de monstration est exhibé (mais aucunement le montage, qui s'affranchit quant à lui du caractère faiblement organisé du film de famille - où les bandes sont simplement mises bout à bout - pour s'inscrire dans une logique narrative), le film désignant Jacques comme l'instance énonciative de son discours. Or ce personnage n'est pas présenté dans les autres parties du film comme étant celui qui filme ce que nous voyons, même lorsqu'il s'agit de sujets caractéristiques d'un film de famille (repas en commun, cérémonie à l'église, discours d'un membre de la famille, etc.). Certes, comme nous le verrons, sa position de "cinéaste» dans les entretiens tend à contaminer la perception que l'on a de son rôle au sein de l'histoire racontée. Ce n'est toutefois pas lui qui la raconte.

\section{Les entretiens, pourvoyeurs d'informants narratifs}

Cette disjonction entre les deux pratiques à l'œuvre dans Mon frère se marie - un dispositif qui rappelle le «cinéma direct» juxtaposé à l'esthétique de la transparence du cinéma traditionnel - ne doit pas laisser croire que les entretiens instaurent une fracture totale avec le déroulement du récit "en acte». En effet, il ne s'agit nullement d'un making of, car les locuteurs et le filmeur - précisons qu'il n'est jamais question de l'équipe de tournage de Jean-Stéphane Bron - ne cessent jamais d'incarner des personnages de fiction. Non seulement ils se réfèrent à des événements qu'ils n'ont jamais vécus (à l'exception de l'acteur interprétant Vinh, qui peut faire intervenir son expérience personnelle ${ }^{\mathbf{1 1}}$ ), mais, à travers l'entretien lui-même, ils créent leur personnage grâce à un jeu sur la voix, les gestes, les postures, les mimiques, etc. Précisons que ces paramètres ne se résument pas au jeu des acteurs: le cadrage et le montage contribuent également à faire le portrait de ces personnages $\mathbf{1 2}$ (fig. 5). Les réponses des parents aux questions de Jacques participent à l'élaboration de leur profil psychologique, définissant ainsi certains principes relationnels qui régissent l'entièreté de l'univers diégétique. En raison de son caractère ramassé sur le plan temporel - tous les événements se

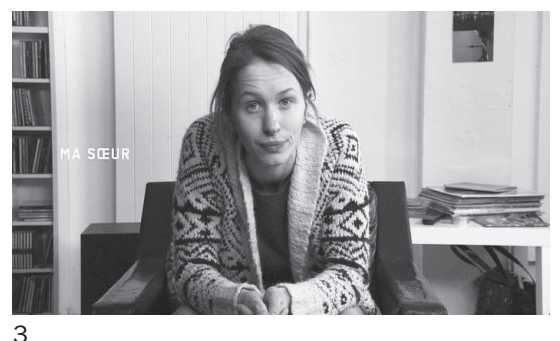

3

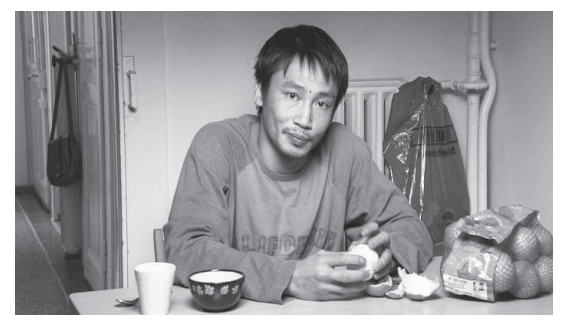

4

11 La présence de cet (unique) acteur non professionnel participe de la logique du film: il est le pivot, le "ferment réel" - il s'agit d'un Vietnamien adopté par des Suisses - autour duquel gravitent les autres personnages, quant à eux définis par le mensonge au sein de la fiction.

12 Ainsi le montage détermine-t-il la durée des prises de parole (le lieu de la coupe finale est fréquemment significatif), l'ordre dans lequel elles apparaissent, les liens qui se tissent avec les plans précédant et suivant l'entretien, etc. A plus large échelle, on repère des phénomènes d'écho témoignant d'une activité configurante située à un niveau énonciatif qui surplombe le jeu des acteurs. Ainsi par exemple du geste qui consiste à éloigner progressivement une main de l'autre en tendant les bras, tel que l'effectuent Vinh, Claire et Catherine dans des contextes différents, puis qui est inversé lorsque Vinh rabat ses mains l'une contre l'autre pour signifier qu'au mariage les deux mères n'en faisaient plus qu'une: ce parcours à travers des fragments d'entretiens disjoints est celui qui va de la séparation à l'union, l'un des principaux sujets du film. 


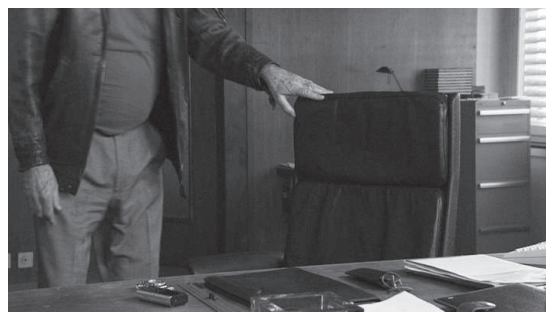

5/ Le visage du père (Jean-Luc Bideau) est initialement rejeté hors-champ, de sorte que la composition du cadre met en évidence le fauteuil du bureau, déplacement métonymique qui suggère d'entrée de jeu combien la fonction professionnelle a primé chez ce personnage sur le devoir de père de famille.

13 Cité dans Joël Jenzer, "De l'autre côté du réel ", Le Nouvelliste, 4.10.2006, p. 37.

14 Ainsi, le chroniqueur du Corriere del Ticino trouve le film "mi-figue, mi-raisin" [«il film non è né carne né pesce"] (op. cit., p. 22), Norbert Creutz précise dans le chapeau de son article que le film "ne séduit qu'à moitié " (op. cit., p. 33), alors qu'on peut lire dans Le Courrier: "En passant de la comédie de situation à la tragi-comédie, Bron affaiblit quelque peu l'ironie décapante de son propos initial" ("Mensonges et réconciliation dans le film de Bron", Le Courrier, 09.08.2006, p. 4). déroulent sur quatre jours -, la diégèse filmique ne pouvait fournir de façon crédible une représentation nuancée des personnages sans recourir à un expédient qui, plus platement, aurait pu consister en des flashbacks audiovisualisés. Ainsi que le déclare le cinéaste, "ces témoignages, ça permet de ressentir [une] évolution, c'est une manière d'approfondir les personnages et de les humaniser» $\mathbf{1 3}$. Le potentiel de "distanciation» que recèle le dispositif convoqué dans les entretiens est par conséquent subordonné aux nécessités de l'identification aux personnages.

Comme les confessions sont situées, ainsi que le spectateur peut l'inférer après quelques minutes de film, dans l'après du mariage, et portent également sur le passé de la famille, les personnages sont saisis à différentes étapes et de diverses manières (en acte, dans l'évocation de souvenirs personnels ou à travers le discours des autres). Cette psychologisation via le mode de l'entretien est l'indice d'une volonté de soumettre le comique (voire le burlesque) qui affleure çà et là aux impératifs du drame : les protagonistes agissants font place à des personnages définis par leurs affects et leurs pensées. L'hétérogénéité générique qui résulte notamment des interruptions pseudo-documentaires n'est d'ailleurs probablement pas étrangère à ce sentiment "d'entre-deux» qu'ont manifesté de façon récurrente les critiques en émettant un jugement mitigé, quand bien même ces derniers ne pointent pas les intermèdes, mais le ton général du film ${ }^{\mathbf{1 4}}$.

Il est vrai qu'en dépit de son intérêt formel et narratif, cette hybridité a des conséquences manifestes (et discutables) sur la représentation des parents vietnamiens qui, contrairement aux membres de la famille romande, n'ont pas droit au mode de l'entretien (alors que la famille suisse alémanique de la mariée passe quasiment à la trappe!). Bien que cette privation soit motivée dans l'histoire du film (ne serait-ce qu'en raison de la barrière linguistique), elle n'en occasionne pas moins une disparité entre les deux familles : alors que la famille d'adoption de Vinh a droit à des moments d'introspection, le couple invité nous apparaît passablement caricatural - du moins en ce qui concerne l'oncle - dès lors que l'on conçoit sa représentation non plus seulement à l'aune des codes propres à la "comédie légère", mais en fonction du drame familial, genre que le dispositif des entretiens contribue à poser. En effet, les parents de Jacques bénéficient d'une plus grande consistance psychologique en se dévoilant lors des interviews et d'une épaisseur supplémentaire conférée par la connotation de documentarité - ils sont sinon plus "réels», du moins plus réalistes que les Vietnamiens.

Du point de vue de la fonction narrative de ces inserts de type documentaire, il faut également considérer les liens tissés entre le récit audiovisuel de l'ensemble du film et les assertions énoncées verbalement 
devant la caméra - dont le contenu, souvent narratif (biographique), n'est jamais visualisé, si ce n'est dans quelques plans (superflus) qui précèdent la mention du titre du film 15 . Dans un premier temps, le spectateur ne peut situer temporellement les intermèdes par rapport au récit du mariage, car les personnages évoquent des faits (l'adoption de Vinh, la séparation du couple, etc.) certes nécessaires à l'intelligibilité du récit filmique, mais antérieurs au point de départ de ce dernier. Les entretiens offrent toutefois des moments ouvertement axés sur la transmission d'informations narratives, ce qui permet de délester partiellement le reste du film de cette fonction. Dès lors, les dialogues, moins contraints à une clarification destinée au spectateur, peuvent se faire plus implicites. Pour les séquences qui ne sont pas organisées sur le mode de l'entretien, le masquage des stratégies communicatives développées par le film envers son spectateur constitue ainsi un gain de réalisme. Ce n'est qu'au neuvième insert que le spectateur comprend que les interviews sont postérieures au jour du mariage, et donc que les temps verbaux passés se réfèrent à la fois à l'époque où la famille était unie et à celle de la mise en scène mensongère - un double rejet dans le passé qui tend à rabattre une époque sur l'autre, en adéquation avec le désir fantasmatique d’une «unité retrouvée» (ou jamais perdue) qui imprègne tout le film. Claire signifie d'ailleurs que cette mascarade lui apparaissait comme une sorte de flash-back lorsqu'elle déclare: "Je me retrouvais dix ans en arrière" (insert $\mathrm{n}^{\circ} 13$ ). Ce présent dans lequel les personnages miment ce qu'ils étaient fait lui-même partie du passé pour les mêmes protagonistes interrogés ultérieurement par Jacques.

\section{A propos du pré-générique}

Parmi les personnages interviewés, la mère, Claire, occupe une place privilégiée. Tout d'abord, c'est elle qui pose le contexte sociohistorique des familles d'accueil helvétiques à l'époque des boat people vietnamiens dans les années 1980, évocation présentée comme la citation de son journal intime de l'époque; ensuite, c'est elle qui précise les relations temporelles, et qui ancre plus rapidement et plus fréquemment son discours dans le «récit premier» du film (voir par exemple l'insert commentatif $\mathrm{n}^{\circ} 17$, première interruption provisoire d'une séquence continue, celle du repas dans le jardin). En outre, et ce n'est pas un hasard, le film commence par elle, plus précisément par une interaction entre elle et son fils. La mère fait naturellement naître le film (dont le fils, lui, crée le «film dans le film»), et annonce l'arrivée de Vinh dans leur vie vingt ans auparavant tout en accompagnant over la première apparition de ce personnage dans le présent de l'image (insert $n^{\circ} 6$ ), comme si elle le
15 L'unique plan consacré au domicile des parents de Vinh au Vietnam montre comment la mère du jeune homme fixait au mur, derrière un petit autel, les cartes postales de montagnes envoyées chaque année par les Suisses. Ostensiblement, cette brève scène de deux plans relève du cliché (à l'instar des vues de Zermatt) et ne présente aucunement, au niveau de son traitement, la dimension (pseudo)documentaire dont bénéficie la présentation de Claire. 
16 Voir Roger Odin, "L'entrée du spectateur dans la fiction", dans Jacques Aumont et JeanLouis Leutrat (éd.), Théorie du film, Albatros, Paris, 1980; André Gardies, Le récit filmique, Hachette, Paris, pp. 44-45; Alain Boillat, La fiction au cinéma, L'Harmattan, Paris, 2001, chapitre II.

17 Au sens de Roger Odin, "Film documentaire, lecture documentarisante", dans Jean-Charles Lyant et Roger Odin (éd.), Cinémas et réalités, CIEREC/Université de Saint-Etienne, SaintEtienne, 1984, pp. 263-277.

18 Notamment dans La bonne conduite (2000), qui repose sur un principe (celui d'une caméra située frontalement au niveau d'un parebrise) identique à celui dont use Abbas Kiarostami dans Ten (2002). Cette similitude témoigne d'une parenté d'intérêt propre à un certain "cinéma d'auteur" qui, dans le cas de Kiarostami, est associé à la fiction. Notons qu'Atom Egoyan recourt également (mais ponctuellement) à une telle caméra miniaturisée et placée à l'avant d'une voiture dans Felicia's Journey (Le voyage de Félicia, 1999); or le réalisateur canadien est probablement l'un de ceux qui s'est intéressé avec le plus de constance à la "fiction familiale" dans son versant le plus noir et traumatique, y compris dans ses liens, également esquissés dans Mon frère se marie, avec la question des origines (arméniennes dans le cas d'Egoyan).

19 Sur cette problématique telle qu'elle se formule dans le premier long métrage de Bron, Le génie helvétique, voir Alain Boillat et Laurent Guido, "Mais im Bundeshuus, un documentaire au service du récit", Décadrages $n^{\circ} 3$, printemps 2004, pp. 86-97. faisait advenir à la fiction. Dans ce "pré-générique», le film exhibe la genèse de l'histoire qu'il raconte. Plus globalement, le récit de Mon frère se marie est structuré sur une évolution des rapports mère-fils et père-fille, les premiers étant privilégiés puisqu'ils concernent plusieurs couples de personnages (Claire et Vinh, Claire et Jacques, Vinh et sa mère biologique), dont le filmeur des entretiens. L'importance de ce lien maternel affirmé dès la partie liminaire transparaît à plusieurs reprises dans la suite du film, notamment par le biais du traitement de l'espace et des regards dans la séquence où les deux familles sont rassemblées dans la villa (fig. 6-11). La rencontre des deux mères qui se réalise à l'occasion du mariage en Suisse ne peut avoir d'équivalent au niveau paternel, puisqu'on nous apprend que le père biologique de Vinh est décédé.

On sait que les débuts de films constituent le lieu par excellence d'un réglage du contrat de lecture passé avec le spectateur ${ }^{\mathbf{1 6}}$. Le prologue de Mon frère se marie tend par contre à biaiser cette orientation dans la mesure où le dispositif de l'entretien semble appeler une lecture documentarisante $\mathbf{1 7}$ alors que nous sommes dans une fiction. Sur un plan intertextuel, on pourrait dire que cette partie inaugurale crée un pont entre ce film fictionnel et la pratique non-fictionnelle antérieure du cinéaste, d'autant que cette dernière se caractérisait par l'établissement d'un dispositif particulier $\mathbf{1 8}$ et par une tendance à narrativiser l'agencement du matériau rassemblé19. En ce sens, le leurre occasionné par l'approche (pseudo)documentaire est particulièrement efficace: lorsqu'on regarde le film de Bron, il est difficile de dissocier «l'effet de réel» qui confère à la fiction toute sa crédibilité de «l'effet documentaire» induit

Dans des vues à la subjectivité marquée en raison d'un premier plan qui obstrue partiellement le champ, nous voyons d'abord Vinh, encore angoissé à l'idée des retrouvailles, observer sa mère à distance (fig. 6-7); plus tard, Claire occupe à son tour cette position de voyeuse, surprenant une discussion entre Vinh et sa mère, le champ/contrechamp entre les deux protagonistes étant médiatisé par le regard de la mère adoptive (fig. 8-11). Le contre-champ sur cette dernière est ensuite filmé depuis l'intérieur de la pièce (fig. 11) dans un plan qui dévoile à droite, fixée sur la porte, une photographie de Vinh enfant qui rappelle celle montrée plein cadre au début du film - elle s'en distingue toutefois, puisque le jeune

homme qui côtoie Vinh n'est plus le même: il semble qu'à l'acteur qui interprète Jacques s'est substituée une image du cinéaste lui-même. Lorsque l'acte du regard est souligné, cette porte constitue également un "seuil" entre la fiction et le documentaire. Le photomontage est (à) l'image de l'hybridité du film. 
par les traits stylistiques des plans d'entretien. La référence au «film de famille» se voit d'ailleurs elle-même reversée plus largement au compte de la catégorie du "documentaire», du fait que les spectateurs qui assistent dans une salle de cinéma à une projection de Mon frère se marie n'associent pas ce qui a été filmé à leur propre vécu. Jean-Pierre Esquenazi propose la distinction suivante: "dans l'effet ‘documentaire`, au lieu que le spectateur soit lui-même la preuve empirique du film comme dans l'effet 'film de famille), c'est la technique cinématographique elle-même qui est créditée de cette preuve.» $\mathbf{2 0}$ On a vu combien, durant les entretiens, la référence appuyée à l'acte de filmer tend à ancrer l'espace-temps représenté dans une pseudo-réalité.

L'hybridité de l'ouverture de Mon fils se marie - à l'instar du magistral prologue d'Hiroshima mon amour (1959), première fiction d'Alain Resnais $\mathbf{2 1}$ - constitue en quelque sorte un rite de passage qui s'applique au cinéaste comme à ceux parmi les spectateurs qui optent pour une lecture auteuriste. En cela, cette introduction connote au travers des liens qu'elle noue avec les autres films du réalisateur une présence auctoriale qui, d'une certaine façon, intervient dans le fonctionnement même de l'univers fictionnel 22. C'est désormais sur ce point que j'aimerais mettre l'accent en montrant comment le rôle de cinéaste conféré au personnage de Jacques lors des entretiens affecte plus généralement l'ensemble du film.

\section{Filmer par procuration. Jacques ou le "Grand Imagier "}

Le rôle de Jacques dans Mon frère se marie ne se réduit pas à celui d'un cinéaste amateur qui, comme il le dit à son père, a emprunté une caméra

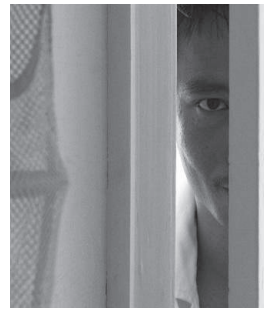

6

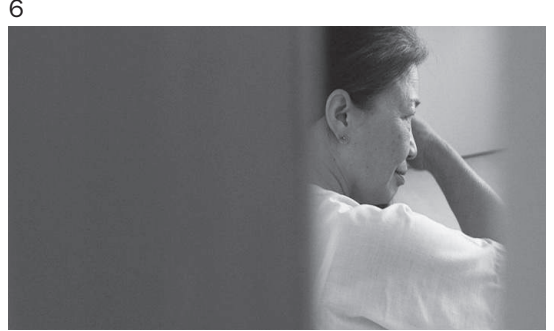

9

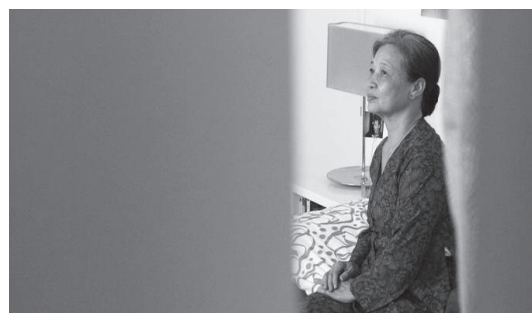

7

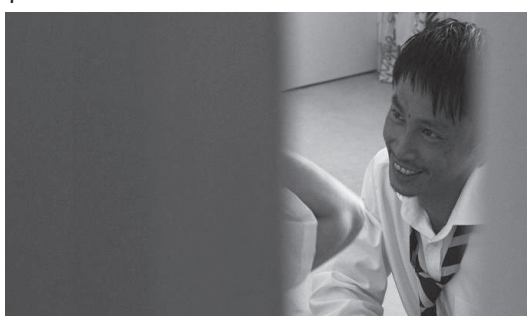

10
20 Jean-Pierre Esquenazi, "L'effet ، film de famille " ", in Roger Odin (éd.), Le Film de famille..., op. cit., p. 212.

21 Remarquons que, chez Alain Resnais, la connotation documentaire ne résulte pas d'une référence au cinéma de type "reportage", mais aux films associant une voix-over homogénéisante et des images d'archives. Alors que le film de Bron impose d'entrée de jeu ses personnages dans leur consistance en les inscrivant dans le hic et nunc de l'entretien, Resnais part d'un sujet parlant désincarné qu'il construit progressivement pour en faire un être de fiction (à propos de ce processus, voir Alain Boillat, Du bonimenteur à la voix-over, Antipodes, Lausanne, à paraître, chapitre 7).

22 Dans la présente analyse, j'ai décidé d'écarter tout lien existant entre la biographie du cinéaste et l'histoire du film pour me concentrer sur la façon dont Mon frère se marie détermine, grâce aux particularités de son organisation discursive, certaines stratégies qui influent sur la façon dont le spectateur appréhende le degré de fictionalité des faits représentés. Toutefois, les correspondances entre la vie de l'auteur et le récit du film ayant été fréquemment notées dans des articles de presse ou des entretiens, ces données paratextuelles interviennent également dans la constitution du "contrat de lecture" établi avec un certain public.

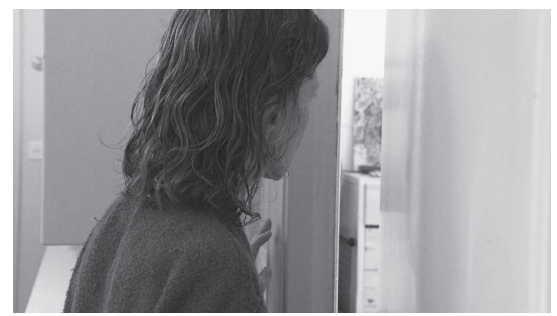

8

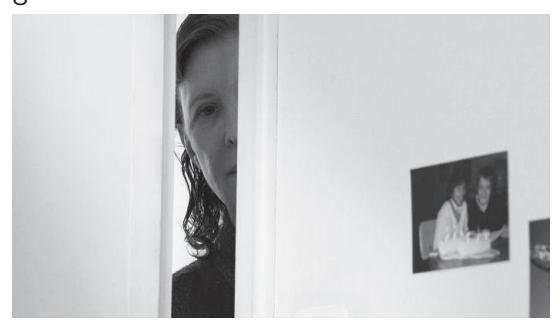

11 


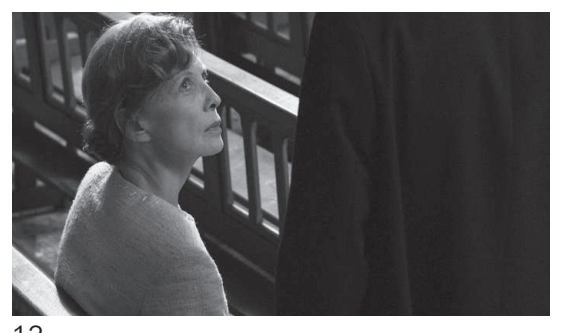

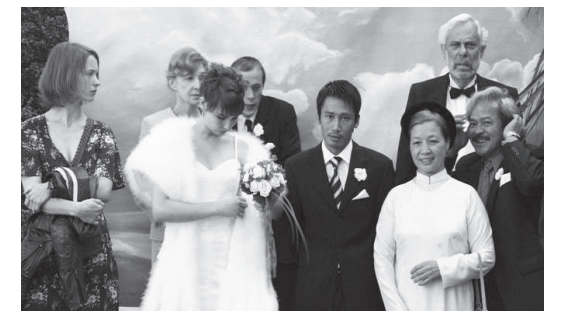

13

sur son lieu de travail pour filmer des entretiens avec les membres de sa famille. Double du cinéaste, son intervention affecte également la "mise en scène» à laquelle s'adonnent les membres de sa famille pour restituer une situation disparue - ressort comique dont (ab)usait notamment, au niveau de l'histoire collective, le film allemand Good Bye, Lenin (Wolfgang Becker, 2003). La maison se fait décor, les gestes du quotidien sont (re)joués. En ce sens, le leurre des entretiens faussement documentaires fait écho à cette "fiction dans la fiction" adressée aux parents de Vinh. Or ce mensonge est orchestré par Jacques, qui, inconsciemment, se sert du mariage comme prétexte pour tenter de ressouder sa famille, comme s'il désirait que cette fiction devienne réalité. L'histoire du film débute en effet par une série d'appels téléphoniques où Jacques informe ses proches de la nécessité de se rassembler à l'occasion du mariage. Cette prise de contact fait en quelque sorte office de casting: Jacques choisit «ses" protagonistes comme il le fait lorsqu'il filme les entretiens, ceux-ci procédant d'une logique de juxtaposition et de variations qui prévaut également dans la série des saynètes de conversation par téléphones portables: différentes réactions face à un événement sont déclinées en fonction des protagonistes. De façon récurrente, Jacques se présente comme l'initiateur ou l'incitateur des actions entreprises par la famille. Inversement, il est construit par le montage comme le destinataire de la mise en scène: ainsi, lorsque tous les membres de la famille sont pris d'un fou rire contagieux, Jacques apparaît en dernier, observant en quelque sorte les fruits de son travail - l'union dans le rire - dans un plan qui clôt significativement la séquence.

Un exemple du rôle d'organisateur endossé par le frère de Vinh me semble révélateur des implications de ce type de personnage sur la question du «film de famille». Il s'agit d'un passage qui fait suite à la cérémonie du mariage, lorsque Claire demeure dans l'église pour s'isoler. A cet instant, c'est précisément Jacques qui vient la chercher et la convainc de rejoindre les autres. Cette séquence est symptomatique des liens existant entre son rôle de "médiateur» pendant la fête - déjà manifeste dans le flash-back du début du film, puisque c'est lui qui apporte à son

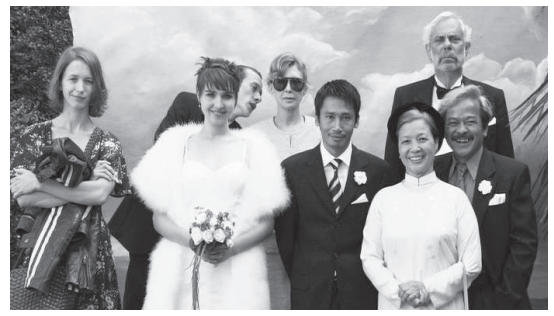

14 
père (qui lui demande des nouvelles de sa sœur) la carte postale écrite par sa mère - et la fonction assignée à ce cinéaste dans le film : Jacques conduit sa mère à l'extérieur pour qu'elle prenne place parmi ceux qui posent pour une photo de groupe, lui indique où elle doit se tenir puis s'approche d'elle et lui demande de sourire (fig. 12-14). On peut dire que, concrètement, il l'introduit dans la future image qui, fixe, est censée représenter de manière emblématique le bonheur familial (alors qu'au moment du déclic, l'image du film fragmente le groupe en isolant les deux invités vietnamiens). La prise de vue à laquelle Claire est incitée à prendre part implique justement une réunion de tous : la «trace» laissée par cet instant est destinée à remplir la fonction traditionnellement dévolue à la photographie de famille, à savoir, ainsi que le notait Pierre Bourdieu, celle de "solenniser et d'éterniser les grands moments de la vie familiale, bref, de renforcer l'intégration du groupe familial en réaffirmant le sentiment qu'il a de lui-même et de son unité»23. En forçant ainsi les choses, Jacques démontre qu'il n'a pas saisi combien sa famille avait perdu conscience de son unité. Par son montage, le début du film participe de cette représentation idéalisée des souvenirs sous forme de photos de famille filmées plein cadre (à la suite des inserts ${ }^{o s} 14$ et 15), qui contrastent avec les images de cartes postales impersonnelles que les Suisses continueront d'envoyer au Vietnam par habitude, mais aussi pour maintenir auprès des parents de Vinh l'illusion d'une pérennité de l'idylle familiale. Ces images sont par contre montrées comme appartenant irrémédiablement au passé, et le film ne se permettra plus aucun flash-back de ce type par la suite.

La place qu'occupe le personnage de Jacques dans le récit de Mon frère se marie permet à mon sens une corrélation entre sa position dans la diégèse - il se situe en quelque sorte "en surplomb" des événements - et sa fonction de cinéaste durant les intermèdes. En effet, ces deux rôles lui confèrent un pouvoir particulier qui, à l'instar de certains narrateurs en voix over ${ }^{\mathbf{2 4}}$, semble le rapprocher du foyer de l'énonciation filmique. Cette "autorité» sur le discours filmique (dans laquelle il ne faut pas seulement voir la création d'un alter ego de l'auteur) n'est bien sûr pas effective, mais résulte elle-même d'une stratégie qui fonde ledit discours, et en cela s'avère productive pour étudier la structure énonciative du film. Bien qu'ancré dans l'univers diégétique, ce personnage s'apparente à cette instance qu'Albert Laffay, dans un texte qui anticipait d'une trentaine d'année la théorie de l'énonciation au cinéma, appelait le "Grand Imagier», c'est-à-dire cette "présence virtuelle cachée derrière tous les films, celle d'une sorte de maître de cérémonie [...] qui donne pour nous aux vues photographiques le sens, le rythme et la durée» 25 . Toutefois, si Jacques
23 Pierre Bourdieu (éd.), Un art moyen. Essai sur les usages sociaux de la photographie, Editions de Minuit, Paris, 1965, p. 39.
24 Sur la question de la corrélation entre le pouvoir octroyé à des personnages au sein de la diégèse et le statut de narrateur over, voir la discussion de thèses issues des gender studies dans le récent ouvrage de Britta Sjogren, Into the Vortex. Female Voice and Paradox in Film, University of Illinois Press, Urbana/ Chicago, 2006.

25 Albert Laffay, "Le récit, le monde et le cinéma", Les Temps modernes, ${ }^{\circ} 21$, mai-juin 1947, p. 1596, repris dans Logique du cinéma, Masson et Cie, Paris, 1964. 
Paris, Texas : l'insertion du film de famille tourné en $8 \mathrm{~mm}$ (fig. 16) instaure une relation de réciprocité entre le père et le fils qui, dans la situation-cadre comme dans l'insert, occupent le même plan, les variations de profondeur de champ créant un aller-retour qui suggère une complicité naissante entre les deux personnages.

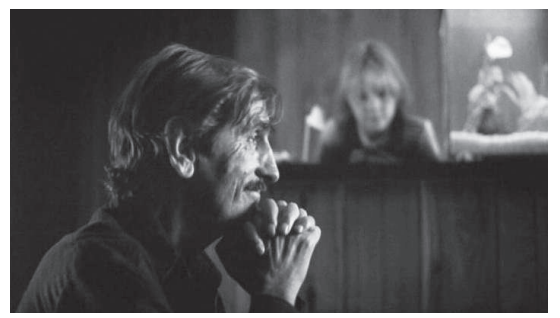

apparaît bien de façon dissimulée comme un "maître de cérémonie» lors du mariage, sa place au "poste de pilotage» (fictif) de l'énonciation filmique dans sa totalité est aussi illusoire que son emprise sur la situation familiale. On l'a dit, le montage des inserts d'entretiens s'effectue "par-dessus son épaule», et toutes les autres images du film existent à son insu. Certes, l'ensemble du film semble placé sous l'emblème de ce personnage en raison de son titre: le possessif "mon» renforce cette impression que le film est une production de Jacques, qu'il en porte en quelque sorte la responsabilité énonciative. En dépit de cet indice péritextuel, elle ne l'est point, sans quoi la fiction se dissoudrait dans le réel.

D’ailleurs, le cours des discussions initiées par Jacques durant les entretiens suggère progressivement que ce personnage perd la maîtrise des événements. Lorsque son père mentionne avec mécontentement le fait que le lieu de la fête a été choisi par Jacques (insert $\mathrm{n}^{\circ} 23$ ), une rupture intervient dans la gestion de ces commentaires rétrospectifs : pendant vingt minutes, ces intermèdes jusque-là réguliers disparaissent totalement. Cette absence s'explique par la nature des événements représentés, qui contredisent l'idéal d'une famille unie secrètement rêvé par Jacques. Le point culminant de ce revirement est la séquence de règlement de compte dans la cuisine, où Jacques doit se confronter physiquement à ses proches. A partir des allocutions tenues devant les invités du mariage, ce sont les autres qui exercent un contrôle discursif. Cette diminution du pouvoir de Jacques sur la cohésion tant filmique que familiale se manifeste également durant les derniers fragments d'entretiens, où les interviewés retournent les questions à l'intéressé avant de donner une réponse ( $\left.\mathrm{n}^{\circ} 25\right)$, voire, en inversant les rôles, l'interrogent sur les motifs qui l'ont poussé à vouloir tout organiser. La distinction entre sujet (le filmeur) et objet (les personnages interviewés) se dissout dans une thérapie familiale interactive. La succession des entretiens s'articule donc elle-même en fonction d'une logique évolutive correspondant à la détérioration des relations entre les membres de la famille, qui s'accroît lorsque le passé s'immisce dans le présent par l'intermédiaire de la chanson (Claire réactive publiquement une habitude d'autrefois). Du
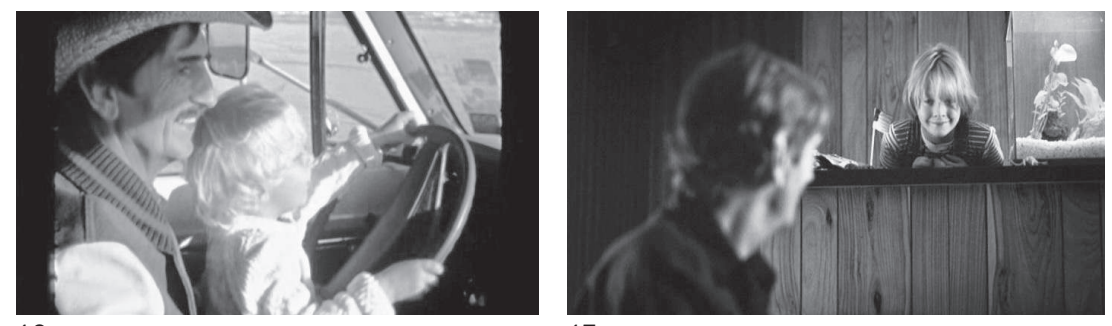

17 
rôle de juge qu'il endossait jusque là, Jacques passe à celui d'accusé. Après avoir tenté de jouer la confession (faussement) "réelle» contre le mensonge (au sein de la fiction), le personnage-cinéaste descend de son piédestal. L'inscription de ce renversement au sein des entretiens prouve à quel point ceux-ci participent pleinement de la structure globale du film, qu'ils révèlent tout en la complexifiant.

\section{Un "film de famille" impossible sans la fiction}

Le point de vue rétrospectif adopté par les protagonistes dans les entretiens marque le récit du sceau de l'échec: l'utopie de la famille unie ne saura, littéralement, trouver son lieu, qu'il s'agisse de la villa ou de la salle des fêtes. C'est d'ailleurs significativement dans des espaces périphériques que les relations se tissent - le père et sa fille au bar, momentanément en retrait par rapport aux festivités - ou que les conflits apparaissent ouvertement. Le renforcement de l'effet de réel auquel concourent les intermèdes permet ainsi d'inscrire une sorte de "retour à la réalité» au sein même de la fiction, le face-à-face avec les protagonistes obligeant le fils à les considérer tels qu'ils sont, non tels qu'il se les imagine. Le recul instauré par le dispositif pseudo-documentaire fait de Mon frère se marie une "fiction familiale» à double titre: il ne s'agit pas seulement d'un récit imaginaire qui aborde le thème de la famille, mais aussi d'une sphère familiale qui éclate au point de ne plus exister qu'en tant que fiction dans l'esprit de Jacques. Comme on l'a vu, la force du film est de rabattre le second niveau (diégétique) sur le premier (filmique) en faisant de Jacques le filmeur des interviews.

Mon frère se marie n'est pas un "film de famille" selon la définition que donnent les théoriciens de cette pratique à la suite de Roger Odin 26, dans la mesure où ce long métrage n'est ni conçu, ni vu comme une réalisation qui requiert le contexte institutionnel spécifique de la sphère familiale, si ce n'est peut-être, en partie, pour la famille réelle de Jean-Stéphane Bron qui voit là, rejoués et fictionnalisés, certains éléments qui la concernent - situations inspirées de la réalité qui ne se résument toutefois pas aux sujets-types des films de famille, censés cristalliser des moments de bonheur idéaux. Mon frère se marie s'apparente cependant à ces fictions cinématographiques étudiées par Marie-Thérèse Journot $\mathbf{2 7}$ qui procèdent à une insertion de fragments documentarisants à l'intérieur de l'univers qu'elles présentent, à l'instar des films d'Atom Egoyan (Next of Kin, 1984, Family Viewing, 1987) ou de Paris, Texas (Wim Wenders, 1984). Toutefois, une différence majeure distingue ceux-ci du film de Bron: Mon frère se marie ne présente pas de mise en scène du visionnement d'un film de famille, mais renvoie au contraire à la phase
26 A l'instar du film amateur, le film de famille constitue en effet un "bon objet" pour l'approche prônée par Roger Odin, la "sémio-pragmatique", qui se définit par la prise en compte conjointe des caractéristiques du texte filmique et du contexte de réception.

27 Marie-Thérèse Journot, "Le film de famille dans le film de fiction", dans Roger Odin (éd.), Le Film de famille..., op. cit., pp. 147-162. 
28 Rappelons que le rapprochement entre le père et le fils, nécessaire à ce récit basé sur la quête de la mère, est signifié dans cette séquence à travers l'organisation des regards par un travail sur la profondeur de champ et le montage. La façon dont Wenders construit cette scène de visionnement - à laquelle il ajoute significativement une musique sirupeuse extradiégétique, et donc imposée par-delà la logique diégétique sur le film de famille muet afin que ce dernier serve pleinement les enjeux de la fiction - renvoie à la fonction sociale de cohésion généralement attribuée au film de famille: l'enfant, qui se refuse d'abord à participer au rituel de la commémoration (il dit à son père adoptif qu'il a déjà vu le film) et fixe obstinément un autre "écran", l'aquarium, noue progressivement une relation au père amnésique à travers la reconnaissance de son propre vécu (et ainsi de leur vécu commun), qu'il pointe dans l'image en s'adressant à Travis ("c'est moi qui conduis", dit-il lorsqu'à l'image Travis le place devant le volant d'un véhicule). Cette réplique se fait l'expression du "phénomène de reconnaissance narcissique" et du "geste désignatif" qui caractérise selon Esquenazi la réception d'un film de famille ("L'effet ، film de famille'", op. cit., pp. 212-213). Ces retrouvailles symboliques entre le père et le fils sont traitées de façon progressive à travers les relations de regards, qui dès lors excluent le spectateur du film pour consolider la cohésion familiale et diégétique.

$\mathbf{2 9}$ Voir la traduction française du manifeste du Dogme par Claire Chatelet, en annexe de son article "Dogme 95: un nouvement ambigu, entre idéalisme et pragmatisme, ironie et sérieux, engagement et opportunisme ", 1895, $n^{\circ} 48$, février 2006, pp. 64-66. du tournage. Les conséquences de cette inversion sont de taille: alors que le pôle de la réception permet la communion des membres autour d'une image(rie) unificatrice qui fait office de flash-back pour le spectateur de la fiction, les entretiens isolent chez Bron chaque parent et n'occasionnent, à quelques rares exceptions près, aucune visualisation du passé. L'adresse à la caméra des personnages interviewés s'oppose à l'instauration, fondée sur les regards, d'un lien entre les personnages de la diégèse, ainsi qu'elle s'effectue par exemple dans la séquence de projection du film 8mm dans Paris, Texas 28 (fig. 15-17). En dépit du ton globalement léger de Mon frère se marie, les intermèdes pseudo-documentaires tendent à offrir, sans atteindre la tension psychodramatique d'un film comme Festen (Thomas Vinterberg, 1998) - dont l'obéissance aux principes du Dogme 9529 induit également une parenté avec le film amateur, sans toutefois que le filmage soit rapporté à une quelconque instance diégétique -, une réflexion pessimiste de (et sur) la famille. A l'hétérogénéité des supports qui caractérise la plupart des fictions intégrant une référence au film de famille (insert d'un film $8 \mathrm{~mm}, 16 \mathrm{~mm}$ ou vidéo dans le standard 35mm), Mon frère se marie présente une image uniformément filmée en vidéo numérique. Jean-Stéphane Bron a su dans ce film exploiter le potentiel associatif lié à cette technique dont l'usage, commun aux sphères privée et professionnelle, permet d'introduire certaines ambiguïtés dans l'organisation énonciative de films comprenant des personnages de «filmeurs» (ou simplement un filmage exhibé, comme dans Garçon stupide de Lionel Baier, 2004). C'est pourquoi les entretiens fictionnels ne sont pas de simples greffes qui mettent à mal la cohérence d'un film dont l'intrigue est elle-même innervée par la tentative d'assimiler dans la «cellule» familiale différents "corps étrangers», mais constituent des contrepoints qui endossent à la fois sur le plan structurel et sémantique une fonction décisive permettant au film d'échapper à la superficialité bonhomme dont on pourrait le gratifier s'il ne recourait pas à certaines stratégies visant à produire un effet documentaire.

\section{Fiche technique}

Réalisation: Jean-Stéphane Bron

Scénario: Jean-Stéphane Bron \& Karine Sudan

Production: Box Productions (Elena Tatti \& Thierry Spicher), Les Films Pelléas (Philippe Martin \& Géraldine Michelot)

Image: Matthieu Poirot-Delpech

Montage: Karine Sudan

Son: Luc Yersin
Interprètes: Aurore Clément (Claire), Jean-Luc Bideau (Michel), Cyril Troley (Jacques), Delphine Chuillot (Catherine), Quoc Dung Nguyen (Vinh), Michèle Rohrbach (Sarah), Man Thu (la mère vietnamienne), Thanh An (Oncle Dac)

Distribution en Suisse: Filmcoopi

Distribution en France: Haut \& Court

Durée: 95 min

Pour plus d'informations, voir www.monfreresemarie.ch/ 


\section{Repérages: les entretiens dans Mon frère se marie}

insert introduit sans retour au récit)

\section{Prologue}

$1^{\text {er }}$ carton de générique

1.) 00 '06"-00'12" (Jacques et Claire)

$2^{\mathrm{e}}$ carton de générique

(Claire over: "Bon alors, j'te regarde ou je regarde dans la caméra?")

2.) 00'18"-00'34" (Claire)

Réponse off de Jacques (visage hors-champ): "Tu me regardes, moi je me mets derrière."

Claire: "Et tu va montrer ça à qui ?"

Jacques: "Juste pour moi."

Mention écrite: "Ma mère"

\section{3.) 00'39"-00'53" (Catherine)}

Mention écrite: "Ma sœur"

Catherine: "Et maman est d'accord?"

Jacques: "Oui... j'ai dit que je ne montrerais cela à personne."

Catherine: "... t'es gonflé!"

Se poursuit over sur un carton de titre: "T'as pas noté les questions quand même!"

\section{4.) 00'59"-01'26" (Michel)}

Mention écrite: "Mon père"

Fin du plan, Jacques (off): "On y va alors?" Michel: "Ouais."

Jacques, over sur un carton de titre: "Pourquoi Vinh est-il arrivé dans la famille?"

5.) 01'31"-01'42" $\rightarrow$ (saute temporelle, identité de cadre):

01'43"-02'11" (Claire)

Ellipse durant le plan, avant que Claire ne lise son journal

6.) 02'12"-02'27" (Vinh, muet; Jacques passe au premier plan devant la caméra)

$\rightarrow$ (continuité sonore), Claire over: "... Vinh arrive enfin..." (alors que l'intéressé prend place dans le cadre)

Mention écrite: "Mon frère"

\section{7.) 02 '28"-03'08" (Claire)}

L'absence de carton entre ce plan et le précédent crée par contraste un effet de coprésence entre Vinh et Claire

8.) 03'09"-03'53" (Michel)

Lien thématique avec ce qui précède (les cartes postales)

Deux plans de flash-back

9.) 04'34"-04'39" (Claire)

"Et chaque année on faisait comme ça, ... jusqu'à ce mariage !"

10.) 04'40"-04'57" (Vinh, Sarah en partie hors-champ)

Mention: "Sarah, ma belle-sœur"

Jacques étant situé dans le hors-champ gauche du cadre, Vinh ne regarde pas la caméra lorsqu'il s'adresse à lui

$\rightarrow$ (Saute temporelle, identité de cadre): 04'57"-05'10" (Vinh)

Fin: "...au paradis"

Générique avec mention des acteurs; fond: images du Cervin (7 plans)

\section{Occurrences ultérieures}

12.) 11'34"-11'53" $\rightarrow$ (saute temporelle minime, identité de cadre) 11'53"-12'05" (Vinh)

13.) 21'21"-21'34" (Claire)

"Je me retrouvais dix ans en arrière, c'était comme si j'avais pas avancé."

$\rightarrow$ 14.) 21'35"-21'54"; 22'03"-22'12" (Catherine)

Inserts de deux photos de vacances (famille à la plage) après "il était pas souvent là lui... sauf pendant les vacances."

$\rightarrow$ 15.) 22'13"-22'23"; 22'30"-22'35" (Vinh)

Inserts de deux photos illustrant le "premier anniversaire en famille (Vinh over sur le premier)

16.) 24'04"-24'33" (Catherine)

Début Jacques off: "Pourquoi tu parlais plus à papa?"

17.) 33'46"-34'15" (Claire)

Début: "Mais je me sentais, mais, complètement à côté de la plaque..."

Inséré durant la séquence du repas sur la terrasse

18.) 45'29"-45'55" $\rightarrow$ (saute temporelle minime, identité de cadre); 45'55"-46'36" (Vinh)

Sujets: la pêche aux coquillages; la fuite en bateau

$\rightarrow$ 19.) 46'37"-47'06" $\rightarrow$ (saute temporelle minime, identité de cadre) 47'06"-47'18" (Claire)

Début: "Ton frère ne nous a pas dit beaucoup de choses de sa vie avant. "; Claire cite ce que la mère de Vinh lui avait dit

20.) 50'57"-51'18" (Vinh)

Début: "Juste avant d'entrer dans l'église, je voyais maman dehors qui faisait les cent pas... C'était comme si je devais choisir."

21.) 52'27"-52'42" (Claire)

A propos des voisins invités pour chanter à l'église

$\rightarrow$ 22.) 57'42"-53'00" (Sarah)

23.) 1 h01'26"-1h01'46" $\rightarrow$ (saute temporelle, identité de cadre) 1h01'46"-1h02'04" (Michel)

24.) $1 \mathrm{~h} 20^{\prime} 09^{\prime \prime}-1 \mathrm{~h} 20^{\prime} 25 "$ (Catherine)

$\rightarrow 25$.) $1 \mathrm{~h}^{2} \mathrm{\prime}^{\prime} \mathbf{2 6}$ "-1h20'40" $\rightarrow$ (saute temporelle minime, identité de cadre); 1h20'40"-1h21'09" (Michel)

Jacques: "Et puis tu te sentais comment? [après la chanson]?

Michel: "Pis toi?"

Jacques: "Ben sonné, mais soulagé aussi."

$\rightarrow 26$.) 1h21'10"-1h21'39" (Claire)

Claire: "Tu voulais nous réunir?"

Jacques: "Mais... non."

Claire: "Mais alors, tu voulais quoi? Tu voulais régler tes comptes?" Jacques: "Non, surtout pas."

Claire (qui semble n'avoir pas entendu): "Comment?"

Jacques: "J'ai dit non, surtout pas."

Claire: "Mais alors quoi?"

Jacques: "Je sais pas. Je voulais qu'on soit tous un peu plus légers." Claire: "Un peu plus légers..." 